\title{
Brain-originated peptides as possible biochemical markers of traumatic brain injury in cerebrospinal fluid post-mortem examination
}

\author{
Mieszko Olczak ${ }^{1}$, Magdalena Kwiatkowska ${ }^{1}$, Justyna Niderla-Bielińska², Dominik Chutorański ${ }^{3}$, Sylwia Tarka ${ }^{1,3}$, \\ Teresa Wierzba-Bobrowicz ${ }^{3}$ \\ ${ }^{1}$ Department of Forensic Medicine, Medical University of Warsaw, ${ }^{2}$ Department of Histology and Embriology, Medical University \\ of Warsaw, ${ }^{3}$ Department of Neuropathology, Institute of Psychiatry and Neurology, Warsaw, Poland
}

\begin{abstract}
The release of brain-originated peptides such as tau protein (MAPT), S-100ß, neurofilament light chain (NFL), and glial fibrillary acidic protein (GFAP) into the cerebrospinal fluid (CSF) has been positively correlated with head injuries in clinical and basic research. In this study, we wanted to examine if selected CSF biomarkers (GFAP, NFL, and myelin basic protein - MBP) of head injury may be useful in post-mortem examination and diagnosis of forensic cases. The study was carried out using cases of head injury and cases of sudden death (cardiopulmonary failure, no injuries of the head as control group) provided by forensic pathologists at the Department of Forensic Medicine, Medical University of Warsaw. Cerebrospinal fluid was collected within $24 \mathrm{~h}$ after death using suboccipital puncture. The concentration of these peptides was compared using an enzyme-linked immunosorbent assay (ELISA). Brain specimens (frontal cortex) were collected during forensic autopsies. Sections were stained immunohistochemically against GFAP, MBP, NF, and amyloid- $\beta$ precursor protein (APP). As a result we documented that elevated levels of CSF, GFAP, MBP, and NFL should be considered a marker for severe and moderate traumatic brain injury. Elevated levels of those peptides combined with a negative APP staining point to their role as markers of head trauma with a shorter time span than APP (manner of minutes).
\end{abstract}

Key words: TBI markers, post-mortem examination, neurofilaments, glial fibrillary acidic protein, myelin basic protein.

\section{Introduction}

Traumatic brain injury (TBI) is among the most frequent neurological disorders worldwide $[24,28]$. Blunt trauma refers to focal brain injury, the action of collision forces to the head and tissue compression in coup and contrecoup mechanisms. Massive acceleration/deceleration of the head results in the emergence of shear, tensile and compressive forces, and diffuse axonal injury (DAI) occurrence [18,31]. More recent studies of moderate and severe TBI revealed both focal lesions and DAI in $50 \%$ of the patients, indicating that the previously described division is artificial, and the term of "traumatic axonal injury" (TAI) is better suited to describe the whole spectrum of axonal lesions [6,27]. Brain injuries should be divided into: primary ones, occurring at the moment of trauma (often termed primary axo- 
tomy), and secondary, delayed and non-mechanical ones (often termed secondary axotomy) referring to factors such as oedema, raised intracranial pressure, cerebral blood flow dysfunction and changes at the cellular level (glutamate excitotoxicity, ionic imbalance, dysfunction of mitochondria, oxidative stress, inflammatory factors release, necrosis, and apoptosis) [12,31]. Clinical diagnosis of TBI includes neurological examination, computed tomography (CT) in the acute phase (due to its high sensitivity to focal lesions) and magnetic resonance imaging (MRI) (T2-weighted imagining, fluid attenuated inversion) in subacute or chronic phases (due to its non-haemorrhagic contusions and oedema superior detection) $[7,8]$. Diffusion tensor imagining (DTI) is a relatively new MRI modality, which seems to be a promising tool for DAl imaging $[15,16]$.

Microtubule associated protein (MAPT) release into the cerebrospinal fluid (CSF) and blood has been interpreted as indicative of axonal injury because its elevated levels were observed in Olympic boxers even after mild head trauma suggesting central nervous system (CNS) injuries [20,21]. The release of other brain-originated peptides, such as calcium-binding protein S-100 $\beta$ (S-100 $\beta$ ), neurofilament light chain (NFL), and glial fibrillary acidic protein (GFAP) into the CSF have also been positively correlated with head injuries $[1,20]$. Neurofilament heavy chain (NFH), tau protein (MAPT), S-100 $\beta$, and myelin basic protein (MBP) serum release were observed in an animal model of TBI with no lesions in macroscopic neuropathological examination [23]. Brain-originated peptides still have considerable diagnostic and therapeutic value because they reflect core elements of the $\mathrm{TBI}$ and supplement functional and imaging-based assessments. Currently the tentative biofluid markers for acute brain injury in the clinical setting include biomarkers of astroglial injury: S-100 $\beta$, GFAP; biomarkers of neuronal injury: neuron specific enolase (NSE), ubiquitin C-terminal hydrolase (UCH-L1); biomarkers of axonal injury: $\alpha$-II-spectrin (A2S), MAPT, and neurofilament heavy (NFH) and light (NFL) chains $[14,17,33]$. Selected potential TBI markers and their sources are illustrated in Figure 1.

Neuropathological microscopic examination of DAI includes visualisation of retraction balls in silver staining (cases with survival over $24 \mathrm{~h}$ ) and amyloid- $\beta$ precursor protein (APP) immunohistochemistry (cases with survival over 3 h) [25]. In our previous study, we documented elevated MAPT serum and CSF levels in post-mortem examinations of $\mathrm{mTBI} / \mathrm{TBI}$ cases with the survival time of minutes after the injury. This finding was accompanied by ventricle wall ruptures, damage to the blood-brain barrier (BBB) cellu-

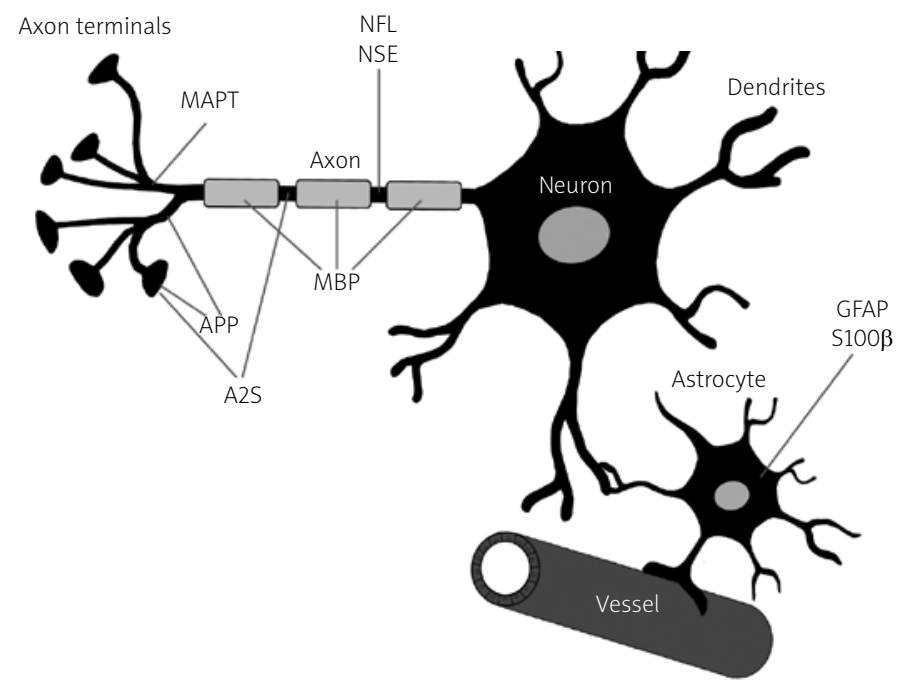

Fig. 1. Selected potential traumatic brain injury markers and their sources: MAPT - tau protein, APP - amyloid- $\beta$ precursor protein, A2S - $\alpha$-II spectrin, MBP - myelin basic protein, NFL - neurofilament light chain, NSE - neuron specific enolase, GFAP - glial fibrillary acidic protein, S-100 $\beta$. 
lar components - rupture of vessels endothelia and astrocyte clasmatodendrosis including astrocyte feet lesions and microglia activation [22]. In this study, we wanted to examine if other CSF biomarkers of head injury could also be useful in post-mortem examination and diagnosis of forensic cases.

\section{Material and methods}

The study was carried out using 21 cases of severe head injury suspected to be the cause of death (contusions, abrasions, or lacerations visible on the skin of the head, deaths not preceded by hospitalisation, nor cardiopulmonary resuscitation - CPR) and 17 cases of sudden death (cardiopulmonary failure - cardiac arrest due to acute myocardial failure with no CPR, no injuries of the head) provided by forensic pathologists at the Department of Forensic Medicine, Medical University of Warsaw. All deaths occurred at the scene of the incident, without medical intervention (CPR). Additional information concerning the circumstances of death was collected from the case files. Study groups were the same cases as presented in our previous publication [22]. The mean age of the deceased was $53.7 \pm 3.9$ years in the experimental group and $55.8 \pm 3.0$ years in the control group. Groups did not differ statistically with respect to age $(p>0.05)$. No signs of neurodegeneration were observed in the neuropathological examination. Also, medical records and family testimony did not report neurodegenerative brain disease history in any of the patients. Control group cases revealed no morphological signs of brain injury on macroscopic or microscopic level. The head injury group revealed blunt injuries to the head (contusions, abrasions, lacerations, haemorrhages in the subcutaneous tissue of the head) on the post-mortem examination. The injuries were followed in some cases with macro and microscopic signs of a brain injury. Some of these traumas (52\% cases, $n=11$ ), were lethal (subdural haemorrhage, brain contusion, intraventricular bleeding). Forty-eight per cent of cases $(n=10)$ revealed minor posttraumatic neuropathological findings, e.g. local subarachnoid haemorrhage, that could not be assumed to be the cause of death. In these cases, the cause of death was multiple trauma (injuries to the head accompanied by lethal injuries to the trunk). Sample brain lesions seen in $\mathrm{HE}$ of the head injury group were presented in our earlier work [22]. Cerebrospinal fluid was collected
12-24 hours after death (prior to the autopsy) using suboccipital puncture.

The skin was decontaminated with $90 \%$ ethanol solution prior to the collection of samples. Samples were immediately centrifuged at $1600 /$ min for $10 \mathrm{~min}$, and supernatant was collected and frozen $\left(-32^{\circ} \mathrm{C}\right)$ for further analysis. Samples with features of haemolysis were excluded from the experiment.

Cerebrospinal fluid GFAP (Millipore, USA cat. No. NS830), NF (Mybiosource, USA, Cat. No. MBS264177), and MBP (Mybiosource, USA, Cat. No. MBS261463) concentrations were measured using double-sandwich ELISA kits according to the manufacturers' protocols. In brief, standards and cerebrospinal samples were incubated in microplate wells precoated with anti-human GFAP, NF, or MBP antibodies, followed by incubation with a biotin-labelled anti-human GFAP, NF, or MBP antibody solution and then incubated with a streptavidin-horseradish peroxidase conjugate. Between each step, the plates were thoroughly washed four times with a washing buffer. After the last washing step, the substrate solution (3,3',5,5'-tetramethylbenzidine) was added. The reaction was stopped by the addition of an acidic solution, and the absorbance of the resulting colour product was measured by reading the ELISA plate at $450 \mathrm{~nm}$. Concentrations of samples were determined using the standard curve. The minimum detectable amount of GFAP was $1.5 \mathrm{ng} / \mathrm{ml}$, NF was $0.06 \mathrm{ng} / \mathrm{ml}$, and MBP was $5 \mathrm{pg} / \mathrm{ml}$, and all concentrations below were reported as undetected. Samples above detection range were diluted, reanalysed, and the results multiplied by the appropriate dilution factor. All samples were assayed in duplicate, and mean \pm SD results were used for analysis.

Brain specimens (frontal lobes) were collected during forensic autopsies and fixed in buffered formalin, then embedded in paraffin. Sections were stained immunohistochemically with anti-MBP (Cell Marque, 1 : 200), anti-NF (MerckMillipore, 1 : 100), anti-GFAP (Serotec, $1:$ 700), and anti-APP (Novus Biologicals, 1 : 250).

The microphotographs of the brain sections were taken with an Olympus BX41 microscope and an Olympus DP25 digital camera.

The STATISTICA software package for Windows (Stat-Soft, Tulsa, OK, USA) was used to analyse all data. Probability $(p)$ levels of less than 0.05 were considered significant. The one-sample Kolmogorov-Smirnov test was used to assess whether the biomarker levels were 
normally distributed. If they were not, the groups were tested for statistically significant differences using the non-parametric Mann-Whitney $U$ test.

\section{Results}

Cases in which head trauma was fatal and those where head injuries did not directly result in death did not differ statistically from each other in the concentration of selected biomarkers $(p>0.05)$. For this reason, both groups were joined and compared to the control group.

Results presented in Figure 2A show an elevated CSF level of MBP in patients with head injury as compared to the control group: $129.78 \pm 79.53 \mathrm{pg} / \mathrm{ml}$ vs. $1356.74 \pm 323.66 \mathrm{pg} / \mathrm{ml}$. Mann-Whitney $U$ test revealed that the CSF-MBP concentration was significantly higher in the head injury group than in the control group $(p<0.001)$. Results presented in Figure 2B show an elevated CSF level of NFL in patients with head injury compared to the control group: 9.87 $\pm 0.52 \mathrm{ng} / \mathrm{ml}$ vs. $41.29 \pm 5.17 \mathrm{ng} / \mathrm{ml}$. Mann-Whitney $U$ test revealed that the CSF-NFL concentration was significantly higher in the head injury group than in the control group $(p<0.001)$. Results presented in Figure $2 \mathrm{C}$ show an elevated CSF level of GFAP in patients with head injury compared to the control group: $201.21 \pm 66.65 \mathrm{ng} / \mathrm{mlvs} .2346 .75 \pm 1312.33 \mathrm{ng} / \mathrm{ml}$. Mann-Whitney $U$ test revealed that the CSF-GFAP

\section{A}

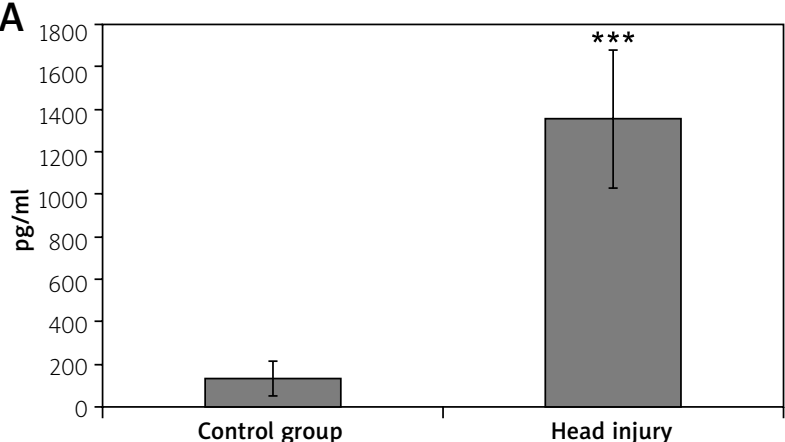

Data shown as mean \pm SEM

* represents $p<0.05,{ }^{* * *}$ represents $p<0.001$

Fig. 2. Biochemical changes in cerebrospinal fluid (CFS) after a head injury. A) Elevated myelin basic protein levels are visible in the CSF in the deceased with a head injury. B) Elevated neurofilament light chain levels are visible in the CSF in the deceased with a head injury. C) Elevated glial fibrillary acidic protein levels are visible in the CSF in the deceased with a head injury. concentration was significantly higher in the head injury group than in the control group ( $p<0.001)$.

To understand the observed changes (elevated MBP, NFL, and GFAP levels in the CSF in the deceased with head injury), additional immunohistochemical reactions against myelin basic protein (MBP), neurofilaments (NF), and astroglia (GFAP) were done to illustrate the potential lesions involving these elements of neurons/ glial cells that were the cause of the observed results. The reaction against amyloid- $\beta$ precursor protein (APP) was done to compare our observations with previously validated methods of axonal injury detection.

The control group presented no lesions. The head injury group presented astrocyte clasmatodendrosis, with astrocyte endfeet damage around the vessels, neurofilament rupture, and diminished reaction to MBP. There were no differences in APP immunostaining between the head injury and control groups (no retraction bulbs were observed). Sample microphotographs are shown in Figure 3.

\section{Discussion}

In our study elevated MBP, NFL, and GFAP levels were observed in the CSF of the deceased from the head injury group irrespective of whether the sustained head injury was fatal (severe) or moderate (not assumed to be the cause of death). Our findings

B
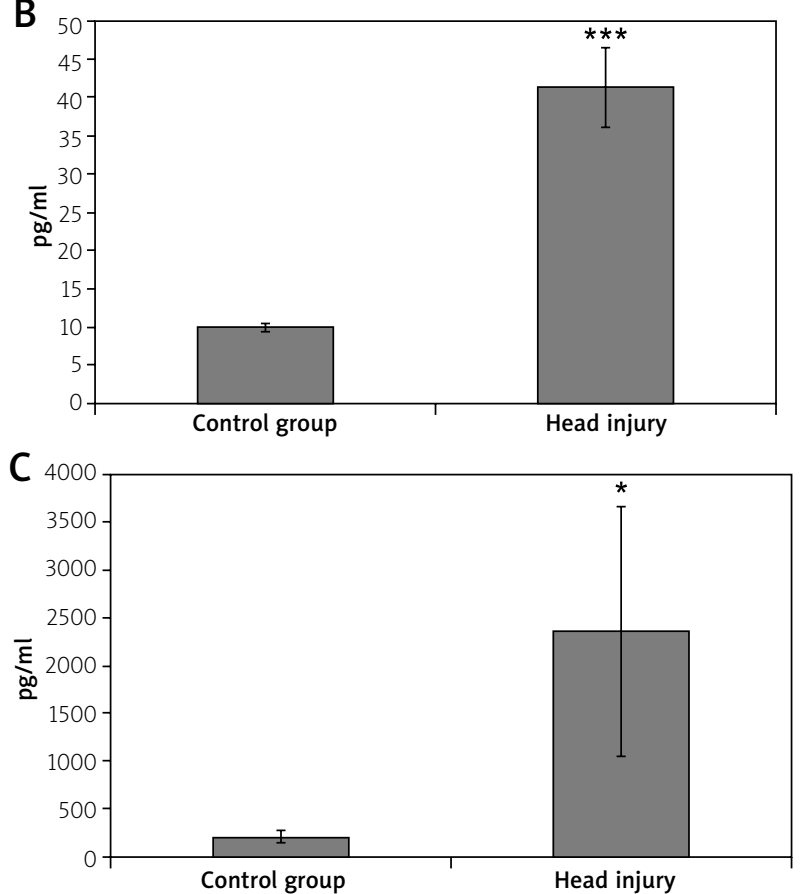


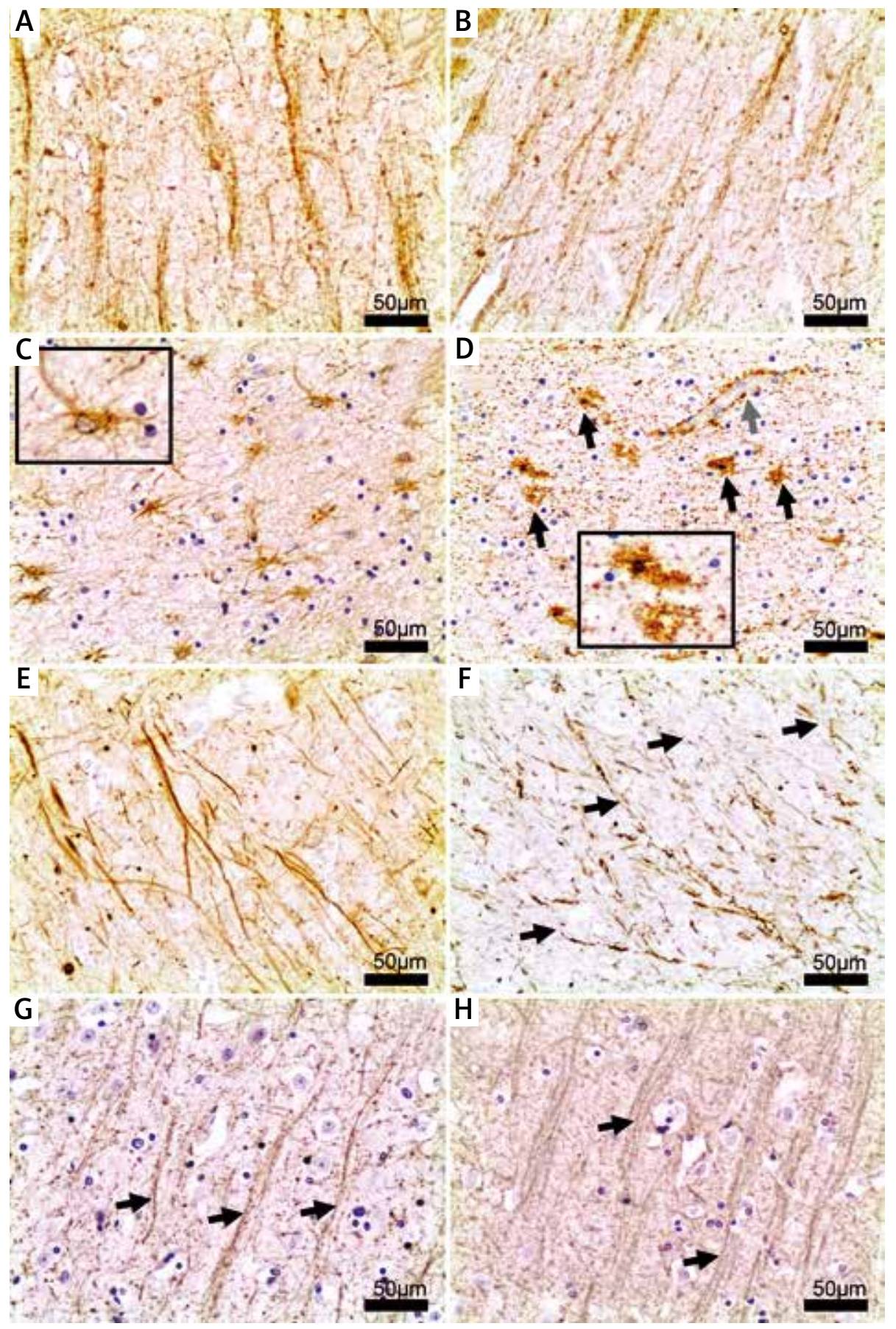

Fig. 3. A) Amyloid- $\beta$ precursor protein (APP) (with no haematoxylin), control group, magnification 200x. B) APP, fatal head injury group, magnification 200x, no retraction bulbs (no differences comparing to control group). C) Glial fibrillary acidic protein (GFAP), control group, magnification 200x, undamaged astrocytes. D) GFAP, fatal head injury group, magnification 200×, astrocyte clasmatodendrosis (black arrow), perivascular astrocyte clasmatodendrosis (grey arrow). E) Neurofilaments (NF), control group, magnification 200x, undamaged neurofilaments. F) NF (with no haematoxylin), fatal head injury group, magnification 200x, ruptured neurofilaments (arrows). G) Myelin basic protein (MBP), control group, magnification 200x, myelin positive reaction (arrows). H) MBP, fatal head injury group, magnification 200x, reduced (minimal) antibody reaction to MBP (arrows). Inserts (C, D) present astroglia in a higher magnification. The number and severity of changes observed in GFAP, NF, and MBP stainings increased with the severity of the head injury sustained. 
are consistent with previous studies on living TBI survivors [30] and animal models of mild TBI [32]. CNS lesions and mechanisms of CNS injury biomarker exit including a direct release (to the CSF) and the action of the glymphatic system were described in our earlier publication. Those lesions included ventricle wall ruptures and astrocyte clasmatodendrosis including periventricular areas, which could be the source of direct exit of CNS biomarkers to the CSF [22]. Because the CSF is in direct contact with the brain ECF, its composition reflects biochemical changes that occur in the brain, including the deeper parts, from which biomarkers may be taken via the glymphatic system to the CSF $[3,10,11]$. Our findings were compared with the results of immunohistochemical stainings and were predictive of neuronal/axonal injury even in moderate TBI cases where the head injury was not assumed to be the cause of death. Increased GFAP, NFL, and MBP CSF level in head injury patients corresponded with frontal lobe disseminated astrocyte clasmatodendrosis, neurofilament rupture, and diminished MBP expression, respectively. Astrocyte clasmatodendrosis and neurofilament rupture may be related to primary and secondary axotomy - due to oedema, raised intracranial pressure, cerebral blood flow dysfunction (ischemia and hypoxia), and as well changes at the cellular level, such as glutamate excitotoxicity, ionic imbalance, dysfunction of mitochondria, oxidative stress, inflammatory factors release, necrosis and apoptosis $[13,29]$. We find the diminished MBP expression which could be a result of secondary processes including intracellular calcium overload from glutamate toxicity or mitochondrial dysfunction and oxidative stress $[2,26]$. Damage to myelin may also cause myelin debris that can stimulate microglial activation [4], which was also described in our earlier publication [22]. Diminished MBP expression and neuroglia (astroglia and oligodendroglia) injury deserve attention because their dysfunction may link head trauma with subsequent demyelination diseases $[5,26]$. The negative result of the APP staining, with no retraction bulbs, is in line with previous studies showing that increased APP levels require longer periods of survival time (at least 35 minutes hours as compared to minutes in our study) $[9,19,25]$. The use of several methods, including immunohistochemical staining, as well biochemical methods of CSF brain-originated peptide determination, can contribute to the more precise dating of the experienced severe and moderate TBI.

\section{Conclusions}

Our study proved that elevated CSF levels of GFAP, MBP, and NFL should be considered as early markers of severe and moderate TBI in post-mortem examination. An increase in levels of these biomarkers, together with the previously reported absence of visible macroscopic traumatic CNS lesions, proves that even mild head injuries result in damage at the neuronal level that could remain undiagnosed during a regular forensic autopsy and routine histopathological examination. The use of several methods, including immunohistochemical staining, as well as biochemical methods of CSF brain-originated peptide determination, can contribute to the more precise assessment of the extent and dating of the experienced severe and moderate TBI.

\section{Disclosure}

The authors report no conflict of interest.

\section{References}

1. Agoston DV, Shutes-David A, Peskind ER. Biofluid biomarkers of traumatic brain injury. Brain Inj 2017; 31: 1195-1203.

2. Benarroch EE. Oligodendrocytes: Susceptibility to injury and involvement in neurologic disease. Neurology 2009; 72: 17791185.

3. Blennow K, Hampel H, Weiner M, Zetterberg H. Cerebrospinal fluid and plasma biomarkers in Alzheimer disease. Nat Rev Neurol 2010; 6: 131-144.

4. Clarner T, Diederichs F, Berger K, Denecke B, Gan L, van der Valk P, Beyer C, Amor S, Kipp M. Myelin debris regulates inflammatory responses in an experimental demyelination animal model and multiple sclerosis lesions. Glia 2012; 60: 1468-1480.

5. Dent KA, Christie KJ, Bye N, Basrai HS, Turbic A, Habgood M, Cate HS, Turnley AM. Oligodendrocyte birth and death following traumatic brain injury in adult mice. PLoS One 2015; 10 : e0121541.

6. Geddes JF, Whitwell HL, Graham DI. Traumatic axonal injury: practical issues for diagnosis in medicolegal cases. Neuropathol Appl Neurobiol 2000; 26: 105-116.

7. Gentry LR, Godersky JC, Thompson B, Dunn VD. Prospective comparative study of intermediate-field MR and CT in the evaluation of closed head trauma. AJR Am J Roentgenol 1988; 150 : 673-682.

8. Hesselink JR, Dowd CF, Healy ME, Hajek P, Baker LL, Luerssen TG. MR imaging of brain contusions: a comparative study with CT. AJR Am J Roentgenol 1988; 150: 1133-1142.

9. Hortobágyi T, Wise S, Hunt N, Cary N, Djurovic V, Fegan-Earl A, Shorrock K, Rouse D, Al-Sarraj S. Traumatic axonal damage in 
the brain can be detected using beta-APP immunohistochemistry within $35 \mathrm{~min}$ after head injury to human adults. Neuropathol Appl Neurobiol 2007; 33: 226-237.

10. Iliff JJ, Wang M, Liao Y, Plogg BA, Peng W, Gundersen GA, Benveniste $H$, Vates GE, Deane $R$, Goldman SA, Nagelhus EA, Nedergaard M. A paravascular pathway facilitates CSF flow through the brain parenchyma and the clearance of interstitial solutes, including amyloid $\beta$. Sci Transl Med 2012; 4: 147ra111.

11. Jessen NA, Munk AS, Lundgaard I, Nedergaard M. The Glymphatic System: A Beginner's Guide. Neurochem Res 2015; 40: 2583 2599.

12. Johnson VE, Stewart W, Smith DH. Axonal pathology in traumatic brain injury. Exp Neurol 2013; 246: 35-43.

13. Kan EM, Ling EA, Lu J. Microenvironment changes in mild traumatic brain injury. Brain Res Bull 2012; 87: 359-372.

14. Kawata K, Liu CY, Merkel SF, Ramirez SH, Tierney RT, Langford D. Blood biomarkers for brain injury: What are we measuring? Neurosci Biobehav Rev 2016; 68: 460-473.

15. Le Bihan D. Looking into the functional architecture of the brain with diffusion MRI. Nat Rev Neurosci 2003; 4: 469-480.

16. Li XY, Feng DF. Diffuse axonal injury: novel insights into detection and treatment. J Clin Neurosci 2009; 16: 614-619.

17. Papa L, Rosenthal K. Biomarkers of Acute Brain Injury in the Emergency Department. In: From Bench to Bedside - Trauma, Tumors, Spine, Functional Neurosurgery. Signorelli F (ed.) InTech 2016

18. Maas Al, Stocchetti N, Bullock R. Moderate and severe trau matic brain injury in adults. Lancet Neurol 2008; 7: 728-741.

19. Morrison C, Mackenzie JM. Axonal injury in head injuries with very short survival times. Neuropathol Appl Neurobiol 2008; 34 124-125.

20. Neselius S, Brisby H, Theodorsson A, Blennow K, Zetterberg H, Marcusson J. CSF-biomarkers in Olympic boxing: diagnosis and effects of repetitive head trauma. PLoS One 2012; 7: e33606.

21. Neselius S, Zetterberg H, Blennow K, Randall J, Wilson D, Marcusson J, Brisby H. Olympic boxing is associated with elevated levels of the neuronal protein tau in plasma. Brain Inj 2013; 27: 425-433.

22. Olczak M, Niderla-Bielińska J, Kwiatkowska M, Samojłowicz D, Tarka S, Wierzba-Bobrowicz T. Tau protein (MAPT) as a possible biochemical marker of traumatic brain injury in postmortem examination. Forensic Sci Int 2017; 280: 1-7.

23. Rostami E, Davidsson J, Ng KC, Lu J, Gyorgy A, Walker J, Wingo D, Plantman S, Bellander BM, Agoston DV, Risling M. A Model for Mild Traumatic Brain Injury that Induces Limited Transient Memory Impairment and Increased Levels of Axon Related Serum Biomarkers. Front Neurol 2012; 3: 115.

24. Rutland-Brown W, Langlois JA, Thomas KE, Xi YL. Incidence of traumatic brain injury in the United States, 2003. J Head Trauma Rehabil 2006; 21: 544-548.

25. Sherriff FE, Bridges LR, Sivaloganathan S. Early detection of axonal injury after human head trauma using immunocytochemistry for beta-amyloid precursor protein. Acta Neuropathol 1994; 87: 55-62.

26. Shi H, Hu X, Leak RK, Shi Y, An C, Suenaga J, Chen J, Gao Y. Demyelination as a rational therapeutic target for ischemic or traumatic brain injury. Exp Neurol 2015; 272: 17-25.
27. Skandsen T, Kvistad KA, Solheim O, Strand IH, Folvik M, Vik A. Prevalence and impact of diffuse axonal injury in patients with moderate and severe head injury: a cohort study of early magnetic resonance imaging findings and 1-year outcome. J Neurosurg 2010; 113: 556-563.

28. Tagliaferri F, Compagnone C, Korsic M, , Servadei F, Kraus J. A systematic review of brain injury epidemiology in Europe. Acta Neurochir 2006; 148: 255-268.

29. Tsitsopoulos PP, Abu Hamdeh S, Marklund N. Current opportunities for clinical monitoring of axonal pathology in traumatic brain injury. Front Neurol 2017; 8: 599.

30. Walder B, Robin X, Rebetez MM, Copin JC, Gasche Y, Sanchez JC, Turck N. The prognostic significance of the serum biomarker heart-fatty acidic binding protein in comparison with s100b in severe traumatic brain injury. J Neurotrauma 2013; 30: 16311637.

31. Werner C, Engelhard K. Pathophysiology of traumatic brain injury. Br J Anaesth 2007; 99: 4-9.

32. Yokobori S, Hosein K, Burks S, Sharma I, Gajavelli S, Bullock R. Biomarkers for the clinical differential diagnosis in traumatic brain injury - a systematic review. CNS Neurosci Ther 2013; 19: 556-565.

33. Zetterberg H, Smith DH, Blennow K. Biomarkers of mild traumatic brain injury in cerebrospinal fluid and blood. Nat Rev Neurol 2013; 9: 201 